\title{
Multimedia Tools to Learn About Nanoparticles Characterization
}

\author{
http://dx.doi.org/10.3991/ijoe.v9iS8.3401 \\ M. G. Rasteiro, A. Cardoso, R. Gomes and T. Santos \\ University of Coimbra, Coimbra, Portugal
}

\begin{abstract}
This work presents the production of multimedia tools for distance learning about nanoparticles characterization. The module is fundamentally based on hypermedia contents and can be included in a course delivered in an eor b-learning format. Hypermedia can include several interactive multimedia contents such as interactive video, hypertext, photos, audio, animations or computer graphics, which can be combined among them. In this work the objective was to develop an interactive video to explain the nanoparticles characterization using dynamic light scattering. This option allows the user to choose the better strategy to learn about the measuring technique, since the video is prepared for interaction and offers the access to complementary information using different hypermedia contents. These aspects are particularly important for lifelong learning courses, dedicated to users already in the job market, for whom the access to real laboratories can be more difficult and restricted.
\end{abstract}

Index Terms-Multimedia tools, interactive videos, distance learning, on-line labs, nanoparticles.

\section{INTRODUCTION}

The methodologies for teaching and learning have been changing, including in Engineering Education, reflecting the evolution in Information and Communications Technology (ICT), the capabilities of the students and the new opportunities for distance learning [1].

New paradigms of learning and training create platforms resourceful in educational contents that are practical, flexible and easy to access, contributing to break down spatial and temporal barriers associated with more traditional educational approaches. In this context, students and trainees can accomplish their own learning process through a laptop, just requiring the availability of an Internet connection to have access to different kinds of multimedia contents and virtual or remote experiments, not only in e/b-learning frameworks, but also in the context of lifelong learning courses or classroom activities [2].

For instance, virtual labs can be a relevant tool to complement real labs: the latter give students "hands-on" exposure, where they can interact with the equipment and know how it operates; the former help prepare the student/trainee to be more productive and effective in the real laboratory. Virtual experiments can include multimedia material for the laboratory visualization of basic phenomena and/or to help the students/trainees to better prepare their lab classes, in order to take better benefit from them [3].

Multimedia contents based on videos of real experiments can play a significant role in this strategy. In general, hypermedia offers a non-linear interactive multime- dia content which can be used as a pedagogical tool, allowing the knowledge discovery and exploration in a constructive and pedagogical approach. Another important aspect is the evaluation of the users' inputs and their behavior during the interaction with the resources and to guide them in order to explore in an enhanced manner either theory or practical contents.

Hypermedia can include different interactive multimedia contents using tools such as interactive video, hypertext, photos, audio, animations or computer graphics, which can be used and combined among them. The contents may be made as resources for pedagogical contexts either to represent or, eventually, control real instruments or virtual ones, or taking part in the experimental process through an environment based on augmented reality. It must be emphasized that each content selection and strategy of presentation must match the unique needs of each user and experiment, using the right medium in the right time, requiring that the contents are self-contained and self-descriptive [4].

Videos of experiments have been previously used by some of the authors of this work for learning purposes, and to prepare students to perform better dealing with real experiments in the laboratory, in a Chemical Engineering course [3].

In this work a module based on multimedia tools was developed for a distance learning course on "Nanoparticles Characterization". Nanoparticles have become increasingly significant, due to their particular and unique properties in various industrial sectors such as pharmaceutical, cosmetics, energy, plastics, aeronautics, space and electronics, among many others. On the other hand, the accurate characterization of their morphology is essential to tune the production and application process but, nowadays, many engineers and technicians in the market lack adequate knowledge in this field.

The module is available online and is essentially based on hypermedia contents, supported on interactive videos, to describe all the steps of the process to characterize the nanoparticles and, to allow the interaction with the user, letting the student/trainee choose which scenes he/she wants to play to learn more deeply each of the different issues. The user can decide if he/she just wants to learn how to apply the measuring technique or if he/she feels the need to go deeper into the learning process about the measuring principles and models in which the characterization technique is based.

The student/trainee can, thus, decide, in each moment, how deeply he/she wants to go into the subject, reexamine the scenes left behind at a later stage, and, therefore, choose which contents are more important for him/her at each instant. 


\section{DESCRITPTION OF THE MODUle CONTENTS}

As referred, the module was prepared to offer the user the possibility to learn about the nanoparticles characterization, in a flexible way and considering different learning contexts. Nanoparticles are very small substances, with a dimension smaller than $10 \mathrm{~nm}$, and it is also possible to take advantage of this feature to obtain their characterization.

The module includes several sections, starting with an introduction to the nanoparticles subject and their applications, and moving, afterwards, to the core of the module which is to learn about the different techniques for nanoparticles characterization. The measurement of the nanoparticles properties such as morphology (size and size distribution, aggregation and shape) and surface energy is addressed. Techniques such as Dynamic Light Scattering (DLS), centrifugation, turbidity analysis, SEM (scanning electron microscopy) and electrophoretic light scattering are tackled during the module. Furthermore, it finishes with a discussion about safety and disposal rules.

To build the module, an interactive video was developed exploring most of the functionalities of popcorn.js library, subtitling, pointing out to new references through twitter, pointing out to papers of reference, all this combined with the classic video production methods, where the script was defined based on the scientific procedure establishing the shot list, hypermedia additional contents and how the information should be presented to the audience.

Videos are one of the most frequently used media in classroom context. However, from a pedagogical point of view, the use of videos as an effective learning medium is subject to some controversy. Still, there are studies [5] referring that videos are only used in broadcast mode. Recent digital forms of video give the student/trainee the opportunity to interact actively with it and control its presentation [6]. One of the drawbacks is that the steady flow of information can lead to a cognitive overload. To avoid this, recipients must be given more control over the flow of information. Interactive videos can help to overcome several of these problems.

For the development of the interactive video we have started with a video on the characterization of nanoparticles, based on the Dynamic Light Scattering - DLS technique. DLS is one of the most used techniques, which takes advantage of the Brownian motion of the nanoparticles, measuring, using an optical technique (light scattering), the particles random velocity resulting from that movement, and relating that velocity with the particles size through the Stokes-Einstein equation.

The entire experiment was filmed first, going from the sample preparation to the nanoparticles characterization, choosing the model for data processing. Subsequently, in order to allow the user to learn more about the characterization process with this video, different hypermedia contents as insertions were introduced during the film (Fig. 1), so that the user can decide which sections to play, depending on his/her interests.

These insertions can be: information about the behavior of the nanoparticles, supplied as text and animations; information about the measurement principles, supplied as text, graphs and schemes; information about data processing procedures and models, supplied as text and graphs and including sometimes the corresponding quanti- tative translation of the models (equations), etc. For example, in the case of the section about nanoparticles properties, the user can find links to other Webpages, text and hypertext, graphs, photos and schemes, for instance, explaining the theory and principles behind DLS and the quantitative models.

The interactive video is divided in five different sections: 1) Introduction, 2) Preparation, 3) Analysis, 4) Results and 5) Conclusion. In section 1, the user can learn about the nanoparticles measurement process, especially using the Dynamic Light Scattering. Section 2 shows the sample preparation for the nanoparticles measurement (Fig. 2) and section 3 describes the procedure to analyze the nanoparticles using the measuring equipment (Fig. 3). In section 4 , the results obtained by applying techniques of data processing are described and presented (Fig. 4). In the last section, some conclusions about the process of nanoparticles characterization using the DLS technique are presented.

Moreover, throughout the video, the user can decide to learn more about some subjects, by clicking on the corresponding menu. In this way, the student/trainee can learn more about: nanoparticles properties or applications; the best way to prepare the sample depending on the material characteristics, including the selection of the adequate concentration; the theory and principles behind DLS; on how to choose the best deconvolution model for data processing, depending on the sample to be characterized; the different types of particle size distribution that DLS can supply; how to measure some of the materials properties required by the measuring technique and which have to be introduced when initiating the measurements (particle and liquid refractive indices and liquid viscosity), etc.

The module available at http://expat.dei.uc.pt/ can be used in courses either for lifelong and distance learning or to undergraduate $\left(1^{\text {st }}\right.$ and $2^{\text {nd }}$ cycles $)$ teaching, for instance in the fields of Chemical, Materials or Biomedical Engineering. Regarding the distance learning course, it will be included in a LMS platform which allows interaction with the users with an intelligent tutoring system [7].

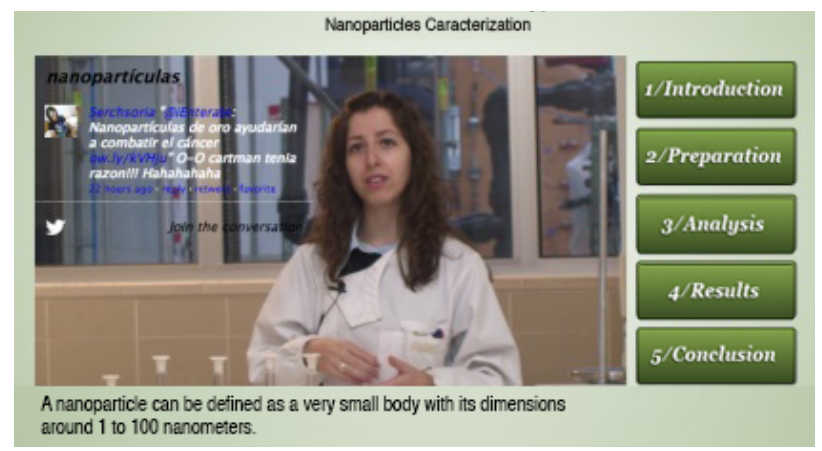

Figure 1. Examples of different hypermedia contents.

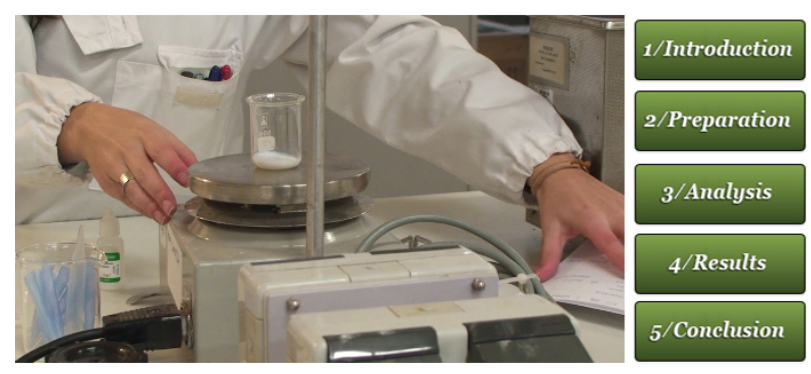

Figure 2. Sample preparation for the nanoparticles measurement. 

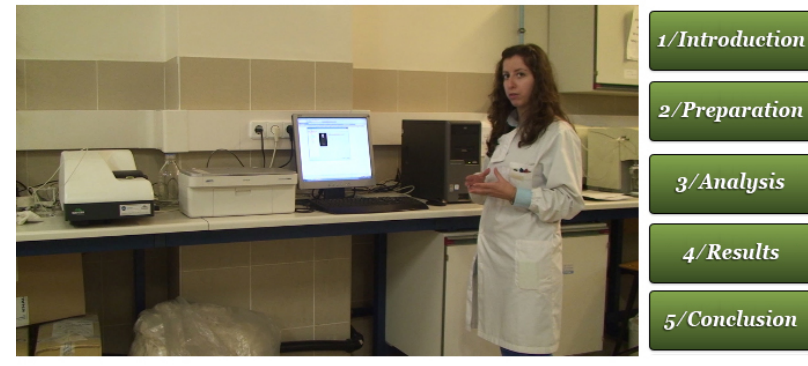

2/Preparation

$3 /$ Analysis

$4 /$ Results

5/Conclusion

Figure 3. Nanoparticles measurement system.

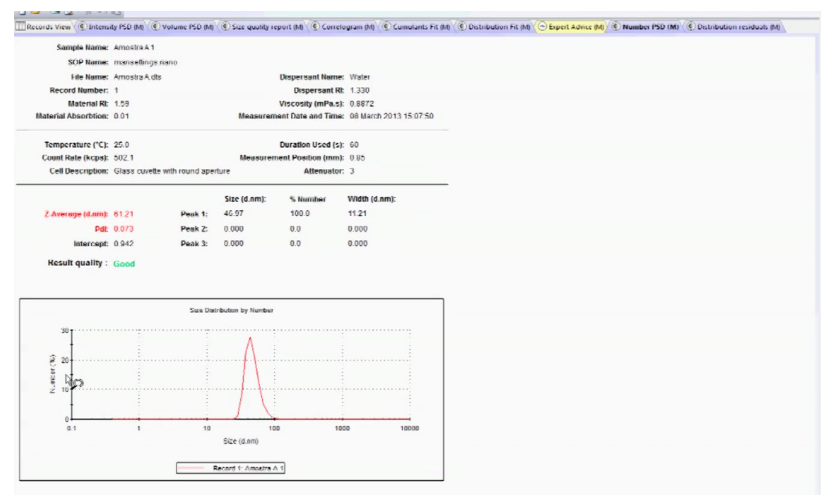

Figure 4. Nanoparticles characterization after data processing.

\section{CONCLUSIONS}

Multimedia tools are of high importance for distance learning courses and when the objective is to train users on some experimental technique, interactive videos are a good option. Hypermedia provides a non-linear interaction with multimedia content, which can be used as a pedagogical tool, allowing the knowledge discovery and exploration in a constructive and pedagogical approach.

This work presents an interactive video for nanoparticles characterization based on the Dynamic Light Scattering technique. It is expected to validate this approach with students of the Chemical Engineering course, in classroom context, and later with the module integration in a distance learning course.

Future work aims to develop more interactive contents with an intelligent and transparent pedagogical guidance, evaluating the users' inputs and reactions to the contents.

This work is included in the wider project Experiment@Portugal'2012.

\section{ACKNOWLEDGMENT}

The authors wish to acknowledge the support provided by Fundação Calouste Gulbenkian (project 122111) experiment@Portugal'2012, as well by FCT (COMPETE, PEst - UI 102 - 2013-2014) for the development of this work.

\section{REFERENCES}

[1] R. M. Felder, "Teaching engineering in the 21 st century with a 12 th-century teaching model: how bright is that?", Chem. Eng. Ed, 40, 2, pp. 110-113, 2006.

[2] Dillenbourg, P., D. Schneider and P. Synteta. Virtual Learning Environments, Proc. of the 3rd Congress on Information and Communication Technologies in Education, Kastaniotis Editions, Greece, 2002, 3-18.

[3] M. G. Rasteiro, L. M. Ferreira, J. C. Teixeira, F. P. Bernardo, M. G. Carvalho, A. Ferreira, R. Q. Ferreira, F. P. Garcia, C. G. Baptista, N. M. Oliveira, M. M. Quina, L. O. Santos, P. A. Saraiva, A. M. Mendes, F. M. Magalhães, A. S. Almeida, J. Granjo, M. Ascenso, R. M. Bastos, R. Borges, "LABVIRTUAL - a virtual platform to teach chemical processes", Education for Chemical Engineers, 4, 1, pp. e9-19, 2009. http://dx.doi.org/10.1016/j.ece.2009. $\underline{02.001}$

[4] N. Pukkhem, W. Vatanawood, "Instructional design using component-based development and learning object classification", 5th IEEE International Conference on Advanced Learning Technologies (ICALT'05), pp. 492-494, 2005. http://dx.doi.org/10.1109/ ICALT.2005.172

[5] J. Stoddard, A. Marcus, "More than showing what happened: exploring the potential of teaching history with film", The High School J., 93, 2, pp. 83-90, 2010. http://dx.doi.org/10.1353/ hsj.0.0044

[6] M. Merkt, S. Weigand, A. Heier, S. Schwan, "Learning with videos vs. learning with print: The role of interactive features", Learning and Instruction, 21, pp. 687-704, 2011.

[7] F. Lecroq, J. Grieu, P. Person, T. Galinho, H. Boukachour, "Inteligent tutoring system in GE3D virtual campus", Int. J. Computer Sc. and Artificial Intelligence, 2, pp. 1-6, 2012. http://dx.doi.org/10.5963/IJCSAI0201001

\section{AuTHORS}

M. G. Rasteiro and R. Gomes are with the Chemical Engineering Department and Research Center for Chemical Processes and Forest Products, University of Coimbra, Pólo II, 3030-790 Coimbra, Portugal (e-mail: mgr@eq.uc.pt, aaritagomes@gmail.com).

A. Cardoso and T. Santos are with the Department of Informatics Engineering, University of Coimbra, Pólo II, 3030-290 Coimbra, Portugal (e-mail: alberto@dei.uc.pt, tiago@tiagosantos.me).

This work was supported by Fundação Calouste Gulbenkian (Projectos Inovadores Domínio Educativo) project 122111 - experiment@Portugal'2012 and by FCT (COMPETE, PEst - UI 102 - 20132014). This article is an extended and modified version of a paper presented at the International Conference exp.at'13, held 18-20 September 2013, in Coimbra, Portugal. Submitted 18 November 2013. Published as re-submitted by the authors 04 December 2013. 